\title{
Statistical mechanics of an ideal Bose gas in a confined geometry
}

\author{
David J. Tom:* \\ School of Mathematics and Statistics, \\ University of Newcastle Upon Tyne, \\ Newcastle Upon Tyne, United Kingdom NE1 $7 R U$
}

(Dated: November 30, 2018)

\begin{abstract}
We study the behaviour of an ideal non-relativistic Bose gas in a three-dimensional space where one of the dimensions is compactified to form a circle. In this case there is no phase transition like that for the case of an infinite volume, nevertheless Bose-Einstein condensation signified by a sudden buildup of particles in the ground state can occur. We use the grand canonical ensemble to study this problem. In particular, the specific heat is evaluated numerically, as well as analytically in certain limits. We show analytically how the familiar result for the specific heat is recovered as we let the size of the circle become large so that the infinite volume limit is approached. We also examine in detail the behaviour of the chemical potential and establish the precise manner in which it approaches zero as the volume becomes large.

PACS numbers: 05.30.Jp,03.75.Hh,03.75.Nt
\end{abstract}

*Electronic address: d.j.toms@newcastle.ac.uk URL: http://www.staff.ncl.ac.uk/d.j.toms/ 


\section{INTRODUCTION}

The study of the ideal Bose gas is a standard topic in any treatment of quantum statistical mechanics. (See Refs. 1, 2, 3, 4] for example.) The usual assumption made is that the bulk, or thermodynamic, limit is taken where the total number of particles and volume of the system are taken to infinity with the particle density fixed. In such a limit, some of the thermodynamic quantities that describe the system, such as the specific heat at constant volume, are not smooth functions of the temperature indicating that a phase transition occurs. This phase transition is usually interpreted as Bose-Einstein condensation of the gas.

If we do not take the bulk limit, by keeping the volume of the system finite, then thermodynamic quantities like the specific heat remain smooth functions of temperature. (This

was emphasized by van Hove [5] for instance.) This smooth behaviour has been borne out in a number of different studies (see the review of Pathria [6] and references therein), and will be shown below. Nevertheless, it seems intuitively plausible that as we let the volume of the system become very large, but still finite, the difference between the non-smooth behaviour in the bulk system and the smooth behaviour in the finite system should become less. The principal aim of this paper is to study in some detail using analytical methods how the bulk limit is approached.

In order to make the calculations as simple as possible we will limit our attention to a three-dimensional space with only one compact dimension. The compact dimension will be chosen to be a circle. We will impose periodic boundary conditions for the circular direction and then study what happens when the radius of the circle tends to infinity. A mathematical technique, used previously to study the Bose gas in a harmonic oscillator potential [7], and a charged Bose gas in a constant magnetic field [8], is used to obtain accurate asymptotic expansions for a number of thermodynamic properties of the system. A short discussion is given at the end of the paper.

\section{THERMODYNAMIC EXPRESSIONS}

We begin with a flat spatial region that is taken to be a finite volume box of dimensions $L, L_{2}, L_{3}$ in the $x, y, z$ directions respectively. The Schrödinger field will be chosen to obey 
periodic boundary conditions on the box walls. We will be interested in the limit where $L_{2}, L_{3} \rightarrow \infty$ so that the spatial region is topologically $\mathbb{S}^{1} \times \mathbb{R}^{2}$.

Under these conditions the energy levels, with $L_{2}, L_{3}$ initially finite, are given by

$$
E_{n n_{2} n_{3}}=\frac{2 \pi^{2} \hbar^{2}}{m}\left(\frac{n^{2}}{L^{2}}+\frac{n_{2}^{2}}{L_{2}^{2}}+\frac{n_{3}^{2}}{L_{3}^{2}}\right)
$$

where $n, n_{2}, n_{3}=0, \pm 1, \pm 2, \ldots$ The total particle number is

$$
N=\sum_{n=-\infty}^{\infty} \sum_{n_{2}=-\infty}^{\infty} \sum_{n_{3}=-\infty}^{\infty}\left[e^{\beta\left(E_{n n_{2} n_{3}}-\mu\right)}-1\right]^{-1}
$$

with $\mu$ the chemical potential and $\beta=T^{-1}$ the inverse temperature (in units where the Boltzmann constant is taken equal to unity). Because the ground state energy vanishes, it is clear that

$$
N_{g r}=\left(e^{-\beta \mu}-1\right)^{-1}
$$

gives the ground state particle number. Bose-Einstein condensation viewed as a phase transition can only occur if $\mu=0$ at some temperature, defined as the critical temperature. A crucial feature of a finite volume system, or a semi-infinite one such as we have here, is that there will be no phase transition characterized by $\mu=0$. Nevertheless, if we view BoseEinstein condensation as defined by a sudden buildup of particles in the ground state over a very small change in temperature, then this can occur even for a finite volume system. An important example of where this happens is for the harmonically confined Bose gas [7, 9]. The principle aim of this paper is to study what happens to the system when the size of the confining volume is varied. In the present setup, this corresponds to changing the circumference of the circle.

It proves convenient to define two dimensionless parameters $x$ and $\epsilon$ by

$$
\begin{aligned}
x & =\frac{2 \pi^{2} \hbar^{2}}{m L^{2}} \beta, \\
\beta \mu & =-\epsilon x .
\end{aligned}
$$

If we let $L_{2}, L_{3}$ become large, then we can approximate the sums over $n_{2}$ and $n_{3}$ that occur in (2.2) with integrals and obtain

$$
N=-L_{2} L_{3}\left(\frac{m}{2 \pi \hbar^{2} \beta}\right) \sum_{n=-\infty}^{\infty} \ln \left[1-e^{-x\left(n^{2}+\epsilon\right)}\right]
$$


The number density is therefore

$$
\rho=\frac{N}{L L_{2} L_{3}}=-\frac{\pi}{L^{3} x} \sum_{n=-\infty}^{\infty} \ln \left[1-e^{-x\left(n^{2}+\epsilon\right)}\right] .
$$

We wish to compare our results for finite $L$ with those found in the bulk, or thermodynamic, limit found when $L \rightarrow \infty$. In this limit we can replace the sum over $n$ with an integral and find the bulk limit to be the standard result of

$$
\rho_{\text {bulk }}=\left(\frac{m}{2 \pi \hbar^{2} \beta}\right)^{3 / 2} \operatorname{Li}_{3 / 2}\left(e^{\beta \mu}\right)
$$

where $\operatorname{Li}_{p}\left(e^{-\theta}\right)=\sum_{n=1}^{\infty} n^{-p} e^{-n \theta}$ is the polylogarithm function. The critical value of $T$ at which $\mu=0$ in the bulk limit will be defined as $T_{0}=\beta_{0}^{-1}$ where

$$
\rho_{\text {bulk }}=\left(\frac{m}{2 \pi \hbar^{2} \beta_{0}}\right)^{3 / 2} \zeta(3 / 2) \text {. }
$$

Even if we keep $L$ finite, so that the bulk approximation is not made, we can still define $T_{0}$ as the temperature at which the density $\rho$ is given by the right hand side of (2.9) . We will define

$$
x_{0}=\frac{2 \pi^{2} \hbar^{2}}{m L^{2}} \beta_{0},
$$

and call $\epsilon_{0}$ the value of $\epsilon$ at the temperature $T_{0}$. Using (2.9) and (2.7) leads to the relation

$$
\pi^{1 / 2} x_{0}^{-3 / 2} x^{1 / 2} \zeta(3 / 2)=-\sum_{n=-\infty}^{\infty} \ln \left[1-e^{-x\left(n^{2}+\epsilon\right)}\right] \text {. }
$$

The point of doing what we have done is that instead of specifying a particle density, we can specify a value for $x_{0}$ instead. The relation (2.11) determines $\epsilon$ as a function of $x$ for a given value of $x_{0}$. By looking at different values of $x_{0}$ we can see how results alter as $L$ is changed. This is completely equivalent to solving for the chemical potential as a function of temperature for a given particle number density, but is better suited to the analytical approximations that we will obtain in the next section.

The result of solving (2.11) numerically for different values of $x_{0}$ is shown in Fig. 11 It can be seen that there is a sharp decrease in $\epsilon$ over a very small range of $x$. From (2.3) with (2.4) this can be seen to correspond to a sudden increase in the number of particles in the ground state, and hence to Bose-Einstein condensation. Note that smaller values of $x_{0}$ correspond to larger values of $L$ from (2.10). As $x_{0}$ decreases, the value of $x$ at which 


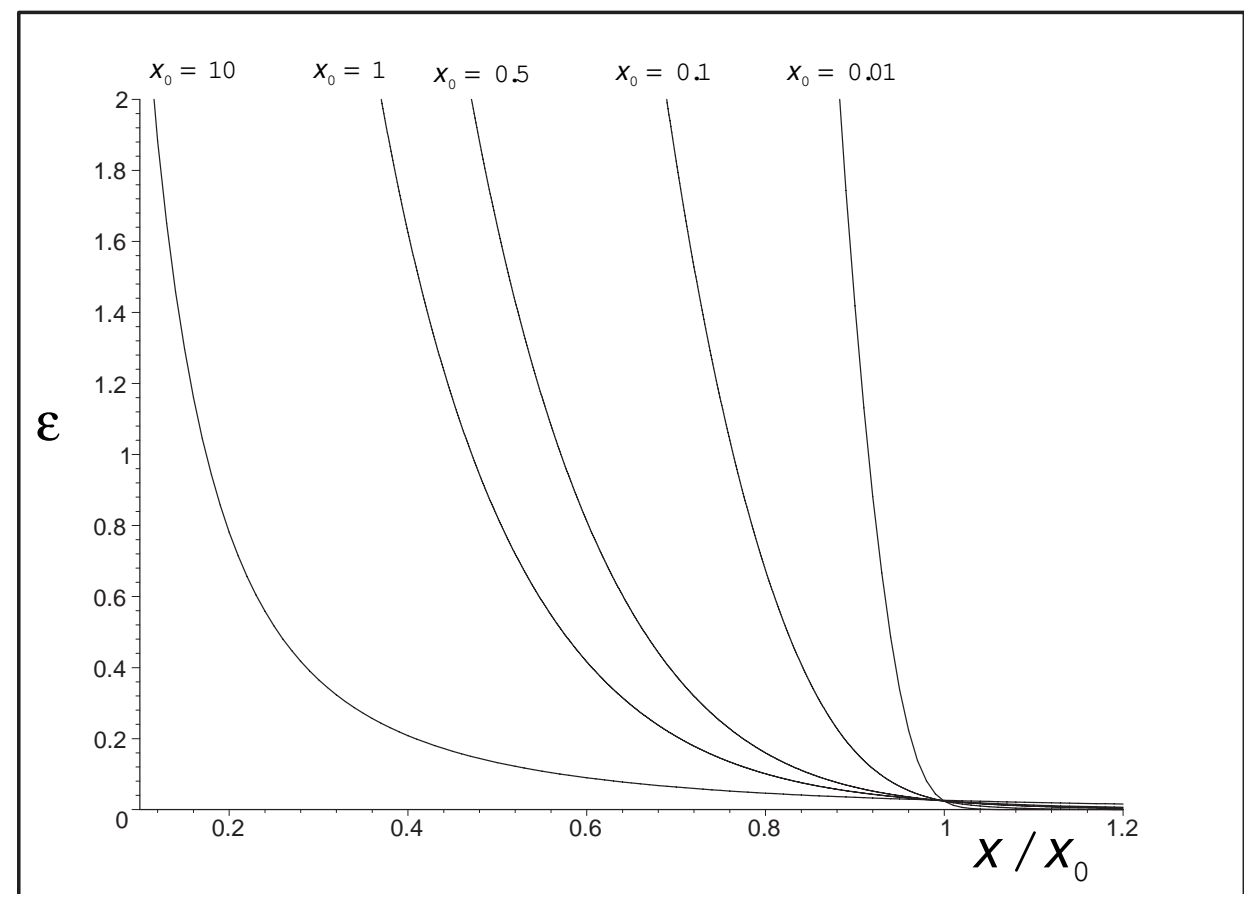

FIG. 1: This shows $\epsilon$ plotted as a function of $x / x_{0}$ for different values of $x_{0}$.

$\epsilon$ undergoes its sudden decrease from values of the order of 1 to values much smaller than 1 , comes closer and closer to the value $x_{0}$. In this sense, as $L$ increases we have a situation that starts to look more and more like a phase transition. We emphasize that there is no true phase transition here because we never attain the value $\epsilon=0$ for any finite value of $L$.

In the bulk limit the specific heat has the familiar peak at the critical temperature $T_{0}$. (See Refs. 1, 2, 3, 4] for example. We also show the result in Fig. 2 for comparison.) We now wish to see what happens when $L$ is finite. The absence of a phase transition in this case indicates that the specific heat should be smooth, unlike the situation for the bulk approximation where the derivative of the specific heat is discontinuous at $T=T_{0}$.

The internal energy is given by

$$
U=\sum_{n, n_{2}, n_{3}} E_{n n_{2} n_{3}}\left[e^{\beta\left(E_{n n_{2} n_{3}}-\mu\right)}-1\right]^{-1} .
$$

Taking $L_{2}, L_{3}$ to be large, and replacing the sums over $n_{2}$ and $n_{3}$ with integrals again leads to

$$
U=-L_{2} L_{3}\left(\frac{\pi}{\beta L^{2}}\right) \sum_{n=-\infty}^{\infty} n^{2} \ln \left[1-e^{-x\left(n^{2}+\epsilon\right)}\right]
$$




$$
+2 L_{2} L_{3}\left(\frac{m}{2 \pi \hbar^{2} \beta^{2}}\right) \sum_{n=-\infty}^{\infty} \operatorname{Li}_{2}\left(e^{-x\left(n^{2}+\epsilon\right)}\right) .
$$

From this result we can compute the specific heat at constant volume from

$$
C_{V}=\left.\left(\frac{\partial U}{\partial T}\right)\right|_{N, V} .
$$

After some calculation, it can be shown that

$$
\frac{C_{V}}{N}=\frac{A}{B}-\frac{C^{2}}{B D}
$$

where

$$
\begin{gathered}
A=\sum_{n=-\infty}^{\infty}\left\{2 \operatorname{Li}_{2}\left(e^{-x\left(n^{2}+\epsilon\right)}\right)-2 x n^{2} \ln \left[1-e^{-x\left(n^{2}+\epsilon\right)}\right]\right. \\
\left.\quad+x^{2} n^{4}\left[e^{x\left(n^{2}+\epsilon\right)}-1\right]^{-1}\right\}, \\
B=-\sum_{n=-\infty}^{\infty} \ln \left[1-e^{-x\left(n^{2}+\epsilon\right)}\right], \\
C=\sum_{n=-\infty}^{\infty}\left\{x n^{2}\left[e^{x\left(n^{2}+\epsilon\right)}-1\right]^{-1}-\ln \left[1-e^{-x\left(n^{2}+\epsilon\right)}\right]\right\}, \\
D=\sum_{n=-\infty}^{\infty}\left[e^{x\left(n^{2}+\epsilon\right)}-1\right]^{-1} .
\end{gathered}
$$

Given the numerical solution for $\epsilon$ found from (2.11), it is a straightforward task to evaluate the sums in (2.16 2.19) and thereby to find the specific heat for given values of $x_{0}$. The results are shown in Fig. 2 for sample values of $x_{0}$. It can be seen that as $x_{0}$ decreases,

corresponding to increasing $L$ and becoming closer to the bulk approximation, the specific heat maximum shifts closer to $x=x_{0}$ and the shape of the curve near the maximum becomes more pointed. This corresponds to what would be expected heuristically, with the bulk approximation becoming indistinguishable from the exact result as $L$ becomes larger.

\section{ANALYTICAL EXPANSIONS}

The key ingredient of the method we will use consists of converting the infinite summations that occur in the expressions of the previous section to a contour integral using the Mellin-Barnes representation for the exponential,

$$
e^{-x}=\frac{1}{2 \pi i} \int_{c-i \infty}^{c+i \infty} d \alpha \Gamma(\alpha) x^{-\alpha}
$$




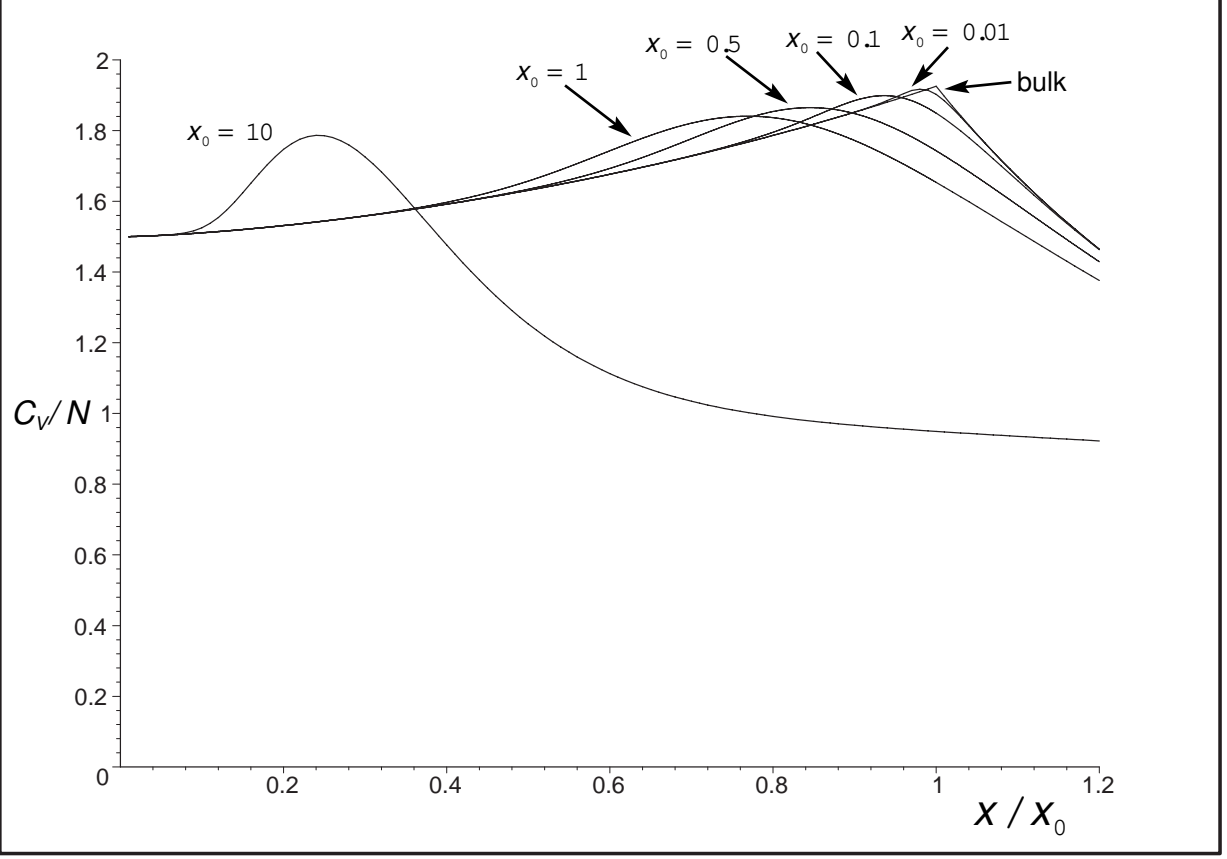

FIG. 2: This shows $C_{V} / N$ plotted as a function of $x / x_{0}$ for different values of $x_{0}$. The result of using the bulk approximation is shown for comparison.

Here $c>0$ is a real constant chosen so that the contour lies to the right of all poles in any of the resulting expressions. The contour is then closed in the left hand of the complex plane and the result evaluated by residues. This method was used previously 7] to obtain analytical expressions for the harmonically confined Bose gas.

We will give a brief description of the method for the sum that occurs in (2.11) and then simply quote the results for the other sums that are needed. Define

$$
L(\epsilon, x)=\sum_{n=-\infty}^{\infty} \ln \left[1-e^{-x\left(n^{2}+\epsilon\right)}\right] .
$$

Start by expanding the logarithm in its Taylor series and then make use of (3.1). This gives

$$
\begin{aligned}
L(\epsilon, x) & =-\sum_{l=1}^{\infty} \frac{1}{l} \sum_{n=-\infty}^{\infty} e^{-l x\left(n^{2}+\epsilon\right)} \\
& =-\frac{1}{2 \pi i} \int_{c-i \infty}^{c+i \infty} d \alpha x^{-\alpha} \zeta(\alpha+1) \Gamma(\alpha) F\left(\alpha, \epsilon^{1 / 2}\right),
\end{aligned}
$$

where we have defined

$$
F\left(\alpha, \epsilon^{1 / 2}\right)=\sum_{n=-\infty}^{\infty}\left(n^{2}+\epsilon\right)^{-\alpha}
$$


The summation in (3.4) converges for $\Re(\alpha)>1 / 2$. We therefore choose $c>1 / 2$ in (3.3) so that all possible poles in the integrand lie to the left of the contour. In the Appendix we show that $\Gamma(\alpha) F\left(\alpha, \epsilon^{1 / 2}\right)$ is an analytic function of $\alpha$ except at $\alpha=1 / 2-n$ for $n=0,1,2, \ldots$ where simple poles occur with residue $\pi^{1 / 2}(-\epsilon)^{n} / n$ !. In particular, $\Gamma(\alpha) F\left(\alpha, \epsilon^{1 / 2}\right)$ is analytic at $\alpha=0$, where $\zeta(\alpha+1)$ has a simple pole with residue 1 . The analytic continuation of $\Gamma(\alpha) F\left(\alpha, \epsilon^{1 / 2}\right)$ to $\alpha=0$ is given in (A7). We therefore find the asymptotic expansion

$$
L(\epsilon, x) \simeq 2 \ln \left[2 \sinh \left(\pi \epsilon^{1 / 2}\right)\right]-\pi^{1 / 2} \sum_{n=0}^{\infty} \frac{(-\epsilon)^{n}}{n !} \zeta\left(\frac{3}{2}-n\right) x^{n-1 / 2} .
$$

An analysis similar to that that we have just described for $L(\epsilon, x)$ may be applied to the other sums that occur in (2.16 2.19). The results are

$$
\begin{aligned}
A \simeq \frac{15}{4} \pi^{1 / 2} \zeta(5 / 2) x^{-1 / 2}-\frac{15}{4} \pi^{1 / 2} \zeta(3 / 2) \epsilon x^{1 / 2} \\
\quad+\epsilon x\left\{4 \ln \left[2 \sinh \left(\pi \epsilon^{1 / 2}\right)\right]+\pi \epsilon^{1 / 2} \operatorname{coth}\left(\pi \epsilon^{1 / 2}\right)\right\} \\
\quad+\frac{15}{4} \pi^{1 / 2} \sum_{n=0}^{\infty} \frac{(-\epsilon)^{n+2}}{(n+2) !} \zeta(1 / 2-n) x^{n+3 / 2}, \\
B=-L(\epsilon, x), \\
C \simeq \frac{3}{2} \pi^{1 / 2} \zeta(3 / 2) x^{-1 / 2}-\pi \epsilon^{1 / 2} \operatorname{coth}\left(\pi \epsilon^{1 / 2}\right)-2 \ln \left[2 \sinh \left(\pi \epsilon^{1 / 2}\right)\right] \\
\quad+\frac{3}{2} \pi^{1 / 2} \sum_{n=0}^{\infty} \frac{(-\epsilon)^{n+1}}{(n+1) !} \zeta(1 / 2-n) x^{n+1 / 2}, \\
D \simeq \pi \epsilon^{-1 / 2} x^{-1} \operatorname{coth}\left(\pi \epsilon^{1 / 2}\right)+\pi^{1 / 2} \sum_{n=0}^{\infty} \frac{(-\epsilon)^{n}}{n !} \zeta(1 / 2-n) x^{n-1 / 2}
\end{aligned}
$$

These results should all be viewed as asymptotic expansions in $\epsilon$ and $x$ that are valid when $\epsilon$ and $x$ are both small. It is straightforward to see that this is the case by comparing the numerical evaluation of the sums in (2.16 2.17) with these analytic expansions. The advantage of the method is that when $x$ is small the direct numerical evaluation of the sums becomes more difficult because they start to become only slowly converging. When $x$ becomes large enough so that the analytical approximations break down (typically $x$ of the order of 0.5 ), the direct evaluation of the sums becomes simple as they converge rapidly.

It is now straightforward to find an expansion for the specific heat using the analytical results of (3.5 3.9) in (2.15). We find, after some calculation,

$$
C_{V} \simeq \frac{15}{4} \frac{\zeta(5 / 2)}{\zeta(3 / 2)}+\alpha_{1} x^{1 / 2}+\alpha_{2} x+\cdots
$$


where the coefficients $\alpha_{1}$ and $\alpha_{2}$ are defined by

$$
\begin{aligned}
& \alpha_{1}= \frac{15}{2 \pi^{1 / 2}} \frac{\zeta(5 / 2)}{\zeta^{2}(3 / 2)} \ln \left[2 \sinh \left(\pi \epsilon^{1 / 2}\right)\right]-\frac{9}{4 \pi^{1 / 2}} \zeta(3 / 2) \epsilon^{1 / 2} \tanh \left(\pi \epsilon^{1 / 2}\right) \\
& \alpha_{2}=\frac{15}{\pi} \frac{\zeta(5 / 2)}{\zeta^{3}(3 / 2)} \ln ^{2}\left[2 \sinh \left(\pi \epsilon^{1 / 2}\right)\right]-\frac{3}{4} \epsilon \\
& \quad+\frac{3}{2 \pi} \epsilon^{1 / 2} \tanh \left(\pi \epsilon^{1 / 2}\right) \ln \left[2 \sinh \left(\pi \epsilon^{1 / 2}\right)\right] \\
& \quad+\frac{15}{4} \frac{\zeta(5 / 2) \zeta(1 / 2)}{\zeta^{2}(3 / 2)} \epsilon+\frac{9}{4 \pi} \zeta(3 / 2) \zeta(1 / 2) \epsilon \tanh ^{2}\left(\pi \epsilon^{1 / 2}\right)
\end{aligned}
$$

Only the first few terms are displayed here. This gives us an analytical approximation to (2.14) that is valid for small $x$ and $\epsilon$. With $T$ fixed, as we let $L \rightarrow \infty$ we have $x \rightarrow 0$ so that only the first term in (3.10) survives. This is the familiar value of the specific heat maximum from the bulk approximation. If we keep $L$ finite, then for small $\epsilon$ it can be shown that $\alpha_{1}<0$, showing that the peak in the specific heat is smaller than that occurring in the bulk limit. This is consistent with what we found numerically as shown in Fig. 2

Using our analytical approximation (3.5) it is possible to obtain an approximate solution to (2.11). This will enable us to study the manner in which the chemical potential vanishes as $L \rightarrow \infty$ and the bulk limit is reached. We have called $\epsilon_{0}$ the value of $\epsilon$ when $x=x_{0}$ (corresponding to the temperature $T=T_{0}$ defined in Eq. (2.10) ). We then have

$$
0 \simeq 2 \ln \left[2 \sinh \left(\pi \epsilon^{1 / 2}\right)\right]+\pi^{1 / 2} \zeta(1 / 2) \epsilon_{0} x_{0}^{1 / 2}+\cdots
$$

for small $x_{0}$ (corresponding to large $L$ ). To lowest order in $x_{0}$ we may ignore the second term in (3.13) and find

$$
\epsilon_{0} \simeq \frac{1}{\pi^{2}} \ln ^{2}\left(\frac{1+\sqrt{5}}{2}\right)
$$

The numerical value of this is $\epsilon_{0} \simeq 0.0234624$, a result that shows up in the numerical evaluation of (2.11) as $x_{0}$ is decreased.

The next order correction to the result in (3.14) will be a term of order $x_{0}^{1 / 2}$. By expanding (3.13) and working consistently to order $x_{0}^{1 / 2}$ it may be shown that

$$
\epsilon_{0} \simeq \frac{1}{\pi^{2}} \ln ^{2}\left(\frac{1+\sqrt{5}}{2}\right)\left\{1-\frac{\zeta(1 / 2)}{\sqrt{5} \pi^{3 / 2}} \ln \left(\frac{1+\sqrt{5}}{2}\right) x_{0}^{1 / 2}\right\} .
$$

In a similar way, more terms in the expansion can be evaluated if desired. If we use (3.15) in (2.5), we can conclude that for large values of $L$ the value of the chemical potential $\mu$ at 
$T=T_{0}$ is,

$$
\mu\left(T=T_{0}\right) \simeq-\frac{2 \hbar^{2}}{m L^{2}} \ln ^{2}\left(\frac{1+\sqrt{5}}{2}\right)\left\{1-\left(\frac{2}{5 \pi m T_{0}}\right)^{1 / 2} \frac{\hbar \zeta(1 / 2)}{L} \ln \left(\frac{1+\sqrt{5}}{2}\right)+\cdots\right\}
$$

The next order term inside the braces is of the order $\left(T_{0} L^{2}\right)^{-1}$. As we let $L \rightarrow \infty$, it is seen that $\mu\left(T=T_{0}\right) \rightarrow 0$ as expected. Our calculation has established in a precise way exactly how the bulk limit is reached.

For temperatures that are close to $T=T_{0}$, a simple extension of the approximate solution to (2.11) that we have presented gives

$$
\epsilon \simeq \frac{1}{\pi^{2}} \ln ^{2}\left(\frac{1+\sqrt{5}}{2}\right)+\frac{\zeta(3 / 2) \ln \left(\frac{1+\sqrt{5}}{2}\right)}{\sqrt{5} \pi^{3 / 2} x_{0}^{1 / 2}}\left[1-\left(\frac{x}{x_{0}}\right)^{3 / 2}\right]
$$

to lowest order. Again it is straightforward to obtain higher order terms in the expansion if needed.

\section{DISCUSSION AND CONCLUSIONS}

We have studied the ideal Bose gas concentrating on the way that the bulk limit is reached. Reliable asymptotic expansions of relevant thermodynamic quantities have been obtained and compared with numerical results. The agreement was good in regions where the asymptotic expansions are expected to be valid. It was shown how the large volume limit of the finite volume system mimics the behaviour of the bulk infinite volume system. With more work it is possible to see how the derivative of the specific heat with respect to temperature becomes discontinuous as the bulk limit is approached.

For simplicity of presentation we have only studied the situation for a 3-dimensional space with one compact circular direction. There is nothing to stop a similar analysis being applied to a space of arbitrary dimension, to more than one compact dimension, or to different boundary conditions. It would also be possible to perform a similar analysis for the relativistic Bose gas and compare with the results of Shiokawa and $\mathrm{Hu}$ [10]. Finally, it would be of interest to study the inclusion of interactions to the analysis. This might be possible using the $\zeta$-function method in Ref. [11]. 


\section{APPENDIX A: SOME SUM RESULTS}

In this Appendix we outline a derivation of the pole structure of $\Gamma(\alpha) F\left(\alpha, \epsilon^{1 / 2}\right)$ using the same methods as in the main text of the paper. We begin by using the standard integral representation 12] for the $\Gamma$-function, assuming initially that $\Re(\alpha)>1 / 2$ so that the sum in (3.4) converges. This gives

$$
\Gamma(\alpha) F\left(\alpha, \epsilon^{1 / 2}\right)=\int_{0}^{\infty} d t t^{\alpha-1} e^{-\epsilon t} \varphi(t),
$$

where

$$
\varphi(t)=\sum_{n=-\infty}^{\infty} e^{-t n^{2}} .
$$

(It can be noted that $\varphi(t)$ is related to the $\theta$-function [12, 13], but we do not use this result here.) Because we assume $\epsilon>0$, and we have $\varphi(t) \rightarrow 1$ as $t \rightarrow \infty$, the integrand of (A1) is well-behaved for large $t$ no matter what the value of $\alpha$. Any poles of $\Gamma(\alpha) F\left(\alpha, \epsilon^{1 / 2}\right)$ must therefore come from the small $t$ behaviour of the integrand.

To get the behaviour of the integrand of (A1) for small $t$, we first use (3.1) to find that

$$
\begin{aligned}
\varphi(t) & =1+2 \sum_{n=1}^{\infty} e^{-t n^{2}} \\
& =1+\frac{1}{i \pi} \int_{c-i \infty}^{c+i \infty} d z \Gamma(z) t^{-z} \zeta(2 z) .
\end{aligned}
$$

The contour in (A3) may be closed noting that there are simple poles at $z=1 / 2$ and $z=0$ only. Evaluating the residues at these poles results in

$$
\varphi(t) \simeq \pi^{1 / 2} t^{-1 / 2}
$$

as $t \rightarrow 0$, a result that agrees with what is found using $\theta$-function identities [12]. If we use $\mathcal{P P}$ to mean the pole part of any expression, then it is clear that

$$
\begin{aligned}
\mathcal{P P}\left\{\Gamma(\alpha) F\left(\alpha, \epsilon^{1 / 2}\right)\right\} & =\mathcal{P} \mathcal{P}\left\{\int_{0}^{1} d t \pi^{1 / 2} t^{\alpha-3 / 2} e^{-\epsilon t}\right\} \\
& =\mathcal{P} \mathcal{P}\left\{\pi^{1 / 2} \sum_{n=0}^{\infty} \frac{(-\epsilon)^{n}}{n !}(\alpha+n-1 / 2)^{-1}\right\} .
\end{aligned}
$$


(In the last line we have simply expanded $e^{-\epsilon t}$ in its Taylor series and integrated the result.) This shows that $\Gamma(\alpha) F\left(\alpha, \epsilon^{1 / 2}\right)$ has simple poles at $\alpha=1 / 2-n$ with residues $\pi^{1 / 2} \frac{(-\epsilon)^{n}}{n !}$ for $n=0,1,2, \ldots$

Although this method is a quick way to uncover the pole terms, if we need to know $\Gamma(\alpha) F\left(\alpha, \epsilon^{1 / 2}\right)$ at values of $\alpha$ where the result is analytic, then a more elaborate analysis must be performed. This has been done by Ghika and Visinescu [14] and in a more general case by Ford [15]. Ford's result gives us

$$
\Gamma(\alpha) F\left(\alpha, \epsilon^{1 / 2}\right)=\pi^{1 / 2} \Gamma\left(\alpha-\frac{1}{2}\right) \epsilon^{1 / 2-\alpha}+4 \Gamma(\alpha) \sin \pi \alpha \int_{\epsilon^{1 / 2}}^{\infty} d x\left(x^{2}-\epsilon\right)^{-\alpha}\left(e^{2 \pi x}-1\right)^{-1}
$$

The poles terms found in (A5) agree with what is found from this exact result. By letting $\alpha \rightarrow 0$ in (A6) it is easily seen that

$$
\left.\left[\Gamma(\alpha) F\left(\alpha, \epsilon^{1 / 2}\right)\right]\right|_{\alpha=0}=-2 \ln \left[2 \sinh \left(\pi \epsilon^{1 / 2}\right)\right]
$$

We also need the value at $\alpha=1$, a result that is easily obtained by noting that

$$
\frac{\partial}{\partial \epsilon}\left[\Gamma(\alpha) F\left(\alpha, \epsilon^{1 / 2}\right)\right]=-\Gamma(\alpha+1) F\left(\alpha+1, \epsilon^{1 / 2}\right) .
$$

(This follows from a straightforward differentiation of (3.4).) Making use of (A7) and letting $\alpha \rightarrow 0$ in (A8) gives

$$
F\left(1, \epsilon^{1 / 2}\right)=\pi \epsilon^{-1 / 2} \operatorname{coth}\left(\pi \epsilon^{1 / 2}\right)
$$

This is a standard result [13] and provides a useful check on the results.

[1] F. London, Superfluids II, (J. Wiley, New York, 1952).

[2] L. Landau and E. M. Lifshitz, Statistical Physics, (Pergammon, London, 1969).

[3] R. K. Pathria, Statistical Mechanics, (Pergammon, London, 1972).

[4] K. Huang, Statistical Mechanics, (J. Wiley, New York, 1987).

[5] L. van Hove, Physica 15, 951 (1949).

[6] R. K. Pathria, Can. J. Phys. 61, 228 (1983).

[7] K. Kirsten and D. J. Toms, Phys. Rev. A 54, 4188 (1996).

[8] G. B. Standen and D. J. Toms, Phys. Lett. A. 239, 401 (1998); Phys. Rev. E 60, 5275 (1999). 
[9] S. Grossmann and M. Holthaus, Phys. Lett. A 208, 188 (1995).

[10] K. Shiokawa and B. L. Hu, Phys. Rev. D 60, 105016 (1999).

[11] D. J. Toms, Phys. Rev. A 66, 013619 (2002).

[12] E. T. Whittaker and G. N. Watson, A Course of Modern Analysis, (Cambridge University Press, Cambridge, 1928).

[13] I. S. Gradsteyn and I. M. Ryzhik, Table of Integrals, Series, and Products, (Academic Press, New York, 1965).

[14] G. Ghika and M. Visinescu, Nuovo Cimento A 46, 25 (1978).

[15] L. H. Ford, Phys. Rev. D 21, 933 (1980). 